\title{
THE N-DIMENSIONAL STERN-BROCOT TREE
}

\author{
Håkan Lennerstad
}

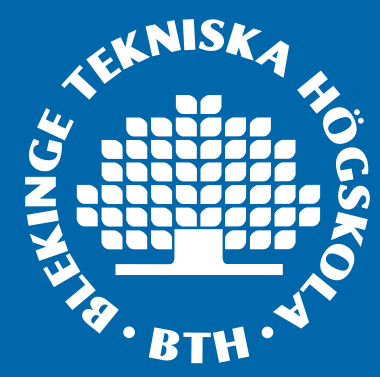




\title{
The $n$-dimensional Stern-Brocot tree
}

\author{
Håkan Lennerstad \\ School of Engineering, Blekinge Institute of Technology, \\ S-371 79 Karlskrona, Sweden \\ Hakan.Lennerstad@bth.se
}

\begin{abstract}
The $n$-dimensional Stern-Brocot tree consists of all sequences $\left(p_{1}\right.$, $\ldots, p_{n}$ ) of positive integers with no common multiple. The relatively prime sequences can be generated branchwise from each other by simple vector summation, starting with an $O N$-base, and controlled by a generalized Euclidean algorithm. The tree induces a multiresolution partition of the first quadrant of the $(n-1)$-dimensional unit sphere, providing a direction approximation property of a sequence by its ancestors. Two matrix representations are introduced, where in both a matrix contains the parents of a sequence. From one of them the isomorphism of a subtree to the entire tree of dimension equal to the number of parents of the top sequence follows.

A form of Fibonacci sequences turn out to be the sequences of fastest growing sums. The construction can be regarded an $n$-dimensional continued fraction, and it may invite further $n$-dimesional number theory.
\end{abstract}

Keywords: Stern-Brocot tree, relatively prime integers, relative primality, Fibonacci numbers, multiresolution partition, ON-base, continued fraction, Minkowski question mark function.

AMS Mathematics Subject Classification: 11A67, 11B75, $11 Z 05$.

\section{Introduction}

The Stern-Brocot tree can be regarded as a tree of all pairs of integers $(p, q)$ that are relatively prime, starting with an ON-base $(1,0)$ and $(1,0)[21]$. It 
is in this paper generalized to the $n$-dimensional Stern-Brocot tree - a tree consisting of all sequences of relatively prime integers $\left(p_{1}, \ldots, p_{n}\right)$, i.e. so that $G C D\left(p_{1}, \ldots, p_{n}\right)=1$. Excempting the ON-base which is the zeroeth generation of the tree, this tree also represents all distinct directions in $\mathbf{Z}_{+}^{n}=$ $\left\{\left(x_{1}, \ldots, x_{n}\right) ; x_{i} \in \mathbf{Z}, x_{i}>0,1 \leq i \leq n\right\}$.

In this introduction, we start by the basics of the two-dimensional tree, proceed with an overview over research in different areas that involves the Stern-Brocot tree, and conclude the section with previous research and main results for the present paper.

\subsection{Basics of the tree}

The mathematician Mouritz Stern and the clockmaker Achille Brocot presented their tree 1858 [34] and 1861 [10], and it has recieved increasing attention since the publication of [21], which is the main reference for the tree and its basic properties. The well known tree (for $n=2$ ) is, except for the first two entries, usually described as a binary tree of ratios in lowest terms as follows:

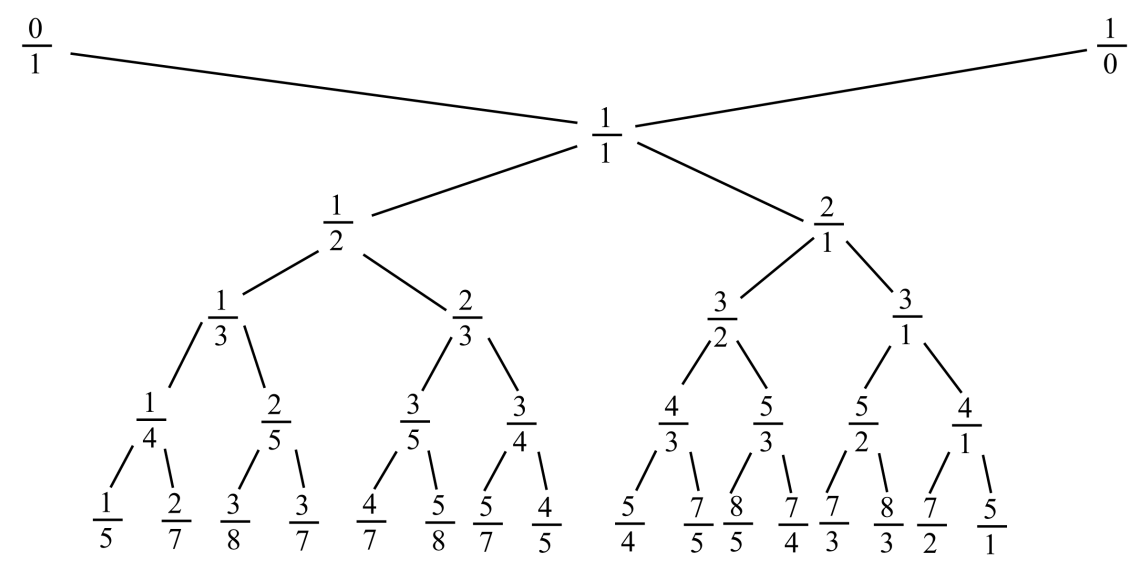

Fig. 1.

This view hints that each ratio has one parent, which is ony appropriate in the matrix description. Each relatively prime pair is constructed generation by generation by vector addition $(a, b)+(c, d)=(a+c, b+d)$, which for ratios 
sometimes is called mediant addition: $\frac{a}{b} \oplus \frac{c}{d}=\frac{a+c}{b+d}[21]$. So $(a+c, b+d)$ appears in an intermediate position between $(a . b)$ and $(c . d)$ in the left-toright increasing size order as ratios. The size order follows from regarding the mediant addition as a weighted mean of $\frac{a}{b}$ and $\frac{c}{d}$ with positive weights: $\frac{a+c}{b+d}=\frac{a}{b} \frac{b}{b+d}+\frac{c}{d} \frac{d}{b+d}$. The algorithm starts with the vectors $(0,1)$ and $(1,0)$ that constitute generation 0 . Thus each entry has two parents, where one parent belongs to the previous generation and the other to an earlier generation.

A pair of parents $(a, b)$ and $(c, d)$ can be characterized by that $c b-a d=1$ assuming $c / d>a / b$ [21], from which it follows that $G C D(a, b)=1$ and $G C D(c, d)=1$. Each pair $(a+c, b+d)$ can also be represented as a ma$\operatorname{trix}\left(\begin{array}{cc}c & a \\ d & b\end{array}\right)$ where the parents are columns. This is a matrix of positive integers of determinant 1 , hence forming a submonoid of the modular group [2], [32]. The representation can be described by a one-to-one function $f:\left(\begin{array}{ll}c & a \\ d & b\end{array}\right) \rightarrow \frac{a+c}{b+d}$. Furthermore, in matrix formulation the generating algorithm can be represented as right-multiplication by the matrices $R=\left(\begin{array}{ll}1 & 1 \\ 0 & 1\end{array}\right)$ and $L=\left(\begin{array}{ll}1 & 0 \\ 1 & 1\end{array}\right)$, the two matrices that constitute the second generation in the tree. In this representation each ratio/pair/matrix has only one parent.

It also follows that each matrix in the tree is a product $R^{a_{0}} L^{a_{1}} \cdot \ldots \cdot R^{a_{n-1}} L^{a_{n}}$ for some integers $a_{0}, \ldots, a_{n}$ which all are positive except $a_{0}$ that may be zero. The product of matrices is equivalent with a continued fraction [21] in the sense that

$$
f\left(R^{a_{0}} L^{a_{1}} \cdot \ldots \cdot R^{a n-1} L^{a_{n}}\right)=a_{0}+\frac{1}{a_{1}+\frac{1}{a_{2}+\frac{1}{\ddots \cdot+\frac{1}{a_{n}}}}}:=\left[a_{0}, \ldots, a_{n}\right],
$$

providing a geometric interpretation of a continued fraction as a walk in the Stern-Brocot tree. This can also be reformulated with the Euclidean algorithm, since if $\frac{a}{b}$ is in lowest terms and $q_{0}, \ldots, q_{n}$ is the sequence of qoutients delivered by the Euclidean algorithm, then

$$
\frac{a}{b}=q_{0}+\frac{1}{q_{1}+\frac{1}{q_{2}+\frac{1}{\cdot \cdot+\frac{1}{q_{n}}}}}
$$


Continued fractions, in [32] connected to geodesics of a modular surface, furthermore provide the best possible approximation of a real number with a rational number that has minimal denominator [21]. In Theorem 15 the $n$-dimensional Stern-Brocot tree is connected to an $n$-dimensional continued fraction.

A Farey series $\mathcal{F}_{n}$ contains all fractions in lowest terms in size order with denominator at most $n$, which is a subtree of the Stern-Brocot tree. As we have seen, a rational number $x \in[0,1]\left(a_{0}=0\right)$ can be mapped onto $L^{a_{1}} \cdot \ldots \cdot R^{a_{n-1}} L^{a_{n}}$ by $f^{-1}$, a mapping that can be extended to irrationals by allowing an infinite expansion. The Minkowski question mark function ? $(x)$ [25] [1] performs this mapping of $x \in[0,1]$ to the $L^{a_{1}} \cdot R^{a_{2}} L^{a_{3}} \cdot \ldots$ expansion in terms of a binary number expansion:

$$
?(x)=a_{0}+2 \sum_{k=1}^{\infty} \frac{(-1)^{k-1}}{\left(a_{1}+\ldots+a_{k}\right)-1} .
$$

The function $?(x)$ is continuous and increasing but has derivative zero in all rational points. It has furthermore a multifractal structure. The formula (3) is due to Denjoy [14].

\subsection{Research areas involving the tree}

We next describe examples of research where the two dimensional SternBrocot tree has turned out to be important, starting with research within mathematics, and later concerning applications. In [4] the authors find algorithms that provide the entry at generation $g$ and position $j$ from the left in the tree, as well as algorithms for the reverse problem. The tree has also been used to find fast algorithms for efficient representation of real numbers on computers [27], [19]. The paper [18] takes an analytic view by building a multifractal analysis for Stern-Brocot intervals, i.e. intervals whose end points are adjacent rationals in the same generation. Analytic properties of the Stern-Brocot pressure function and the multifractal spectrum are studied also together with corresponding results for continued fractions. In [17] the classical Diophantine quadruple problem is adressed. The paper involves a generalisation from $2 \times 2$ matrices to similar $4 \times 3$ matrices, which are defined in terms of the Diothantine problem. The solutions of a modular Diophantine inequality $a x \bmod b \leq c x$ are in [11] proven to be equivalent to finding the right Bézout sequences, which turn out to be specific paths in 
the Stern-Brocot tree. The Gauss map $1 / x-\lfloor 1 / x\rfloor$ acts as a shift operator for a continued fraction. In [5], relations between clusters of this map and branches of the Stern-Brocot tree are proved. Also, a generalised tree is given including also negative rationals, and results for a Gauss map that is enlarged outside the unit interval.

The growth of random Fibonacci sequences, $t_{n+2}= \pm t_{n+1} \pm t_{n}, t_{0}=t_{1}=0$ was in [38] found to be 1.13198824.. with probablity 1, the Viswanath's constant, to be compared to the golden ratio $1.61803398 \ldots$ in the non-random case. It was found by finding a probability measure that is invariant over the division of the real line in Stern-Brocot intervals. This was later generalised in [15] to the random recurrence $t_{n+2}=t_{n+1} \pm \beta t_{n}$, Such random sequences has applications in ergodic theory, dynamical systeems, heavytailed statistics, spectral theory and condensed matter physics. A central observation in these papers is that Stern-Brocot intervals are mapped onto Stern-Brocot intervals by a map that resembles a dilation equation, which in turn is fundamental for the construction of wavelets [35]. Wavelets are sets of real functions with compact support that are orthogonal and ususally display fractal characteristics - supporting a so called multiresolution analysis. They are based on a multiresolution partition, in [36] used for analysis of earthquake data, that is needed in this paper (Definition 1).

In applications, the Stern-Brocot tree has been used not only for the design of cog wheels, which was the start [10]. The two-dimensional tree has been used in discrete geometry and applications, such as studies of digital straight lines - an area of application prone to benefit from a generalization to tree dimensions. One such is presented in [37], for straight segment recognition in discrete linear algebra. Here evolution of slopes corresponds to walks in the Stern-Brocot tree, meaning among other things that that these operations are related to the mediant addition. This addition turned out to be an important tool in the proofs in [23] for comparing ratios, delivering tight bounds on the Euclidean minimal distance valid for any phase shift keying block code with given code size, alphabet size and word length. In [6] the combínatorics of sets of words with certain periodicity properties is examined with the aid of the Stern-Brocot tree, inducing corresponding trees consisting of words. The mapping of $\frac{a}{b}$ to the sequence $a_{0}, \ldots, a_{n}$, defined by $R^{a_{0}} L^{a_{1}} \cdot \ldots \cdot R^{a_{n-1}} L^{a_{n}}$, is sometimes called the Stern-Brocot transform, and has been proposed for string matching problems in security systems [20].

Dynamics of mixed-mode oscillators are since long studied in signal process- 
ing, and are relevant in chemistry and neuroscience. The oscillatory patterns are in [16] shown to be organized efficiently by the tree. The paper [8] implements a finite difference equation for solving the non-linear first order differential Hamilton-Jacobi-Bellman equation that is fundamental in statistic control. The two dimesional problem is studied, which can be formulated as a linear programming problem that is solved by means of the Stern-Brocot tree.

Finally we mention two examples from music theory. In [28] correspondences between the elements of well-formed scales and properties of the modular group and the Stern-Brocot tree are investigated. This provides among other things a language for a discussion about the interface between sound perception and musical thought. The article [12] discusses how Farey series and the Minkowski question mark function emerge in music theory from pitch perception perspectives as well as from musical scale approaches.

\subsection{Main results and related research}

The purpose of $[24]$ was to, for a ratio $a / b$ in lowest terms, find sets

$$
\left\{a_{1} / b_{1}, a_{2} / b_{2}, \ldots, a_{c} / b_{c}\right\}
$$

where $\sum a_{i}=a$ and $\sum b_{i}=b$, and which for fixed $c$ maximizes

$$
\left\lfloor\begin{array}{l}
a \\
b
\end{array}\right\rfloor_{c}=\min \left\{a_{1} / b_{1}, a_{2} / b_{2}, \ldots, a_{c} / b_{c}\right\}
$$

and minimizes

$$
\left\lceil\begin{array}{l}
a \\
b
\end{array}\right\rceil_{c}=\max \left\{a_{1} / b_{1}, a_{2} / b_{2}, \ldots, a_{c} / b_{c}\right\} .
$$

These functions are generalizations of the ceiling and floor functions, which they reduce to when $b=c$. The problem was solved using the (2-dimensional) Stern-Brocot tree. It turned out that the same set $\left\{a_{1} / b_{1}, a_{2} / b_{2}, \ldots, a_{c} / b_{c}\right\}$ provides both the maximum and the minimum, and that it contains at most tree distinct entries, which are parents to each other in the tree. This result allows a solution to a parallel computer performance optimization problem [22], which is a generalized solution to [7], which in turn is a generalization of the famous $4 / 3$-conjecture in computer sciene [13]. 
The usual way to find a ratio $a / b$ in the two dimensional tree is by binary search [21], i.e. by exploiting the left to right size order. In [24] a different algorithm is used, starting with an ON-base $(0,1)$ and $(1,0)$, and writing and rewriting as

$$
\begin{aligned}
(a, b) & =a(1,0)+b(0,1)(\text { start }) \\
x(p, q)+y(r, s) & = \begin{cases}x(p+r, q+s)+(y-x)(r, s) & \text { if } x<y \\
(x-y)(p, q)+y(p+r, q+s) & \text { if } x>y\end{cases}
\end{aligned}
$$

Thus the vectors are descending in the Stern-Brocot tree, while the factors $x$ and $y$ perform a weak Euclidean algorithm: with a quotient that always is one (see page 12). This algorithm is easily generalized from pairs to $n$ tuples (Definition 1), starting the iteration with an $n$-dimensional ON-base. Thus the $n$-dimensional tree can be generated branchwise (Section 2) by a very simple algorithm. The algorithm provides a well defined tree since the conic hull of the sets of vectors produced are disjoint successive refinements of parts of the unit sphere $\mathbf{S}^{n-1}$ or $\mathbf{R}^{n}$, which is appropriately described as a multiresolution partition (Definition 5 and Theorem 1). In the remainder of the paper the tree is described without factors, i.e. generationwise rather than branchwise. A side result of the approach is a generalization of the Euclidean algorithm from 2 to $n$ integers (Definition 3 and Lemma 2).

A difference from the two-dimensional tree is that not all sequences have the same number of parents. The degree $d$ of a sequence is its number of parents: $2 \leq d \leq n$. The conic hull of the sets of parents are $d$-1-dimensional subsets of $\mathbf{S}^{n-1}$ that are rapidly shrinking, meaning that the parents provide relatively primes sequences that approximate the direction of $\left(p_{1}, \ldots, p_{n}\right)$, measured simply as the angle between two vectors (Theorem 4). These are effective approximations partly because of the simplicity of the algorithm, inviting discrete geometry applications.

A $n \times n$-matrix representation (Section 3) $M$ of a sequence of degree $d$ need to be associated with a partition of the columns in $d$ blocks, meaning that the parents are the column block sums. With this matrix representation the fact that all sequences generated by the algoritm are relatively prime can be proved (Theorem 8). Furthemore if follows that all sets of factors are relatively prime (Lemma 9). Also, each sequence/matrix can be seen as a certain product of the matrices of generation 2 (there are $2^{n}-2$ such), just as in the case $n=2$. However, only a certain subset of size $2^{d}-2$ is allowed when having $d$ parents (Theorem 11). 
It is easy to observe that the sequence of matrices produced by the algorithm is a subsequence of an $n$-dimensional continued fraction along $\left(p_{1}, \ldots, p_{n}\right)$, following Brentjes formalism [31]. It also follows that Definition 1 is an $n$-dimensional continued fraction algorithm that is subtractive but not a division algorithm. In [31] convergence issues are studied for multidmensional continued fractions, and they are used among other things for Diophantine approximation.

In a second matrix representation each sequence is associated with a $n \times d$ matrix where the columns simply are the parents. With this matrix representation it can be proven by using the Moore-Penrose pseudoinverse that each subtree, consisting of all descendants of a sequence $\left(p_{1}, \ldots, p_{n}\right)$ of degree $d$, is topologically isomorphic to the entire $d$-dimensional Stern-Brocot tree (Theorem 12).

For convenience we may refer to the subset of the tree that has degree $n$ by the core, while its complement is the pheriphery. The core is an $n$-ary tree with $n^{g-1}$ members in generation $g$. Some basic combinatorics of the tree are given in Section 3.1. Sequences of Fibonacci type (see Definition 4) turn out to have the largest sequence sum in each generation. It is also possible to calculate a formula for $T_{n}(g)$, i.e. the number of sequences of generation $g$ in the $n$-dimensional tree (Theorem 13). Denoting by $T_{n, n}(g)=n^{g-1}$ the number of elements in the core and by $T_{n, F i}(g)=\frac{n !}{(n-g+1) !}$ the number of Fibonacci type numbers, we have the following quantities:

$$
\begin{array}{lllll} 
& n=2 & n=3 & n=4 & n=5 \\
g=2 & 2(2,2) & 6(3,3) & 14(4,4) & 30(5,5) \\
g=3 & 4(4,2) & 24(9,6) & 100(16,12) & 360(25,20) \\
g=4 & 8(8,2) & 84(27,6) & 560(64,24) & 3060(125,60) \\
g=5 & 16(16,2) & 276(81,6) & 2776(256,24) & 21780(625,120) \\
g=6 & 32(32,2) & 876(243,6) & 12824(1024,24) & 139500(3125,120)
\end{array}
$$

Table 1. Values of $T_{n}(g)\left(T_{n, n}(g), T_{n, F i}(g)\right)$ for $g \leq 6$ and $n \leq 5$.

Since $T_{n}(g)$ increases rapidly in both $n$ and $g$, it is natural to consider the Stern-Brocot hedge, which is the significantly smaller subtree of only nondecreasing sequences. For the sake of the generating algorithm this tree only entails different starting sequences - non-decreasing such. When starting the algorithm with at least two members of an ON-base, an not necessarily $n$ members, the algorithm produces the Stern-Brocot grove. It contains $\left(\begin{array}{l}n \\ k\end{array}\right)$ 
copies of the tree of dimension $k \leq n$. The grove contains all distinct directions in $\mathbf{Z}_{+, 0}^{n}=\left\{\left(x_{1}, \ldots, x_{n}\right) ;, x_{i} \in \mathbf{Z}, x_{i} \geq 0,1 \leq i \leq n\right\}$. In the case $n=2$, the hedge is half the tree, and the tree, the grove and the core all coincide.

Section 2 describes the main algorithm and its immediate consequences. Here the framework multiresolution partition is formulated with which the structure of the tree can be understood, and from which the well-definedness of the tree follows. In Section 3 the two matrix descriptions are constructed and used for basic results. Section 3.1 provides basic combinatorics of the $n$-dimensional tree, and Section 4 shows the connection to $n$-dimensional continued fractions, summarizes the results, and formulates further questions.

\section{The generating algorithm}

\subsection{An example}

We start by an example for $n=3$ by calculating the anchestors for the prime triple $(16,9,6)$. Some definitions for general dimensions are made in this subsection.By $m(p, q, r)$ we denote $m$ copies of the vector $(p, q, r)$, and we refer to $m$ as the factor of $(p, q, r)$. The algorithm starts with an $O N$-base $16(1,0,0), 9(0,1,0), 6(0,0,1)$.

In the algorithm, all entries with the smallest factor $m$ are replaced by the sum of all entries, simultaneously decreasing all other factors by $m$. It turns out that the algorithm necessarily ends with the sequence $(16,9,6)$, and that it contains only relatively prime sequences. For the tree structure induced by this algorithm, the parents of a sequence is the sequences that it is the sum of. In some cases two or more entries are least frequent, in which case the cardinality of the number of parents permanently decreases.

Step 0: 16(1,0,0), 9(0,1,0), 6(0,0,1).

Step 1: 10(1,0,0),3(0,1,0),6(1,1,1).

Step 2: $7(1,0,0), 3(2,2,1), 3(1,1,1)$.

Step 3: $4(1,0,0), 3(4,3,2)$.

Step 4: $1(1,0,0), 3(5,3,2)$.

Step 5: 1(6,3,2), 2(5,3,2).

Step 6: 1(11,6,4), 1(5,3,2).

Step 7: $(16,9,6)$. 
The generation number $g(p, q, r)$ is simply the number of steps required by the algorithm to reach $(p, q, r)$, so $g(16,9,6)=7$. In the list above, step $i$ displays the ancestor set $B_{i}(16,9,6)$, for $i=0, \ldots, 7$, i.e. the sets of parent for the sum appearing in the next step. The set $B(16,9,6)=\cup_{i=0}^{7} B_{i}(16,9,6)$ contains all anchestors of $(16,9,6)$, and we can observe that $|B(p, q, r)|=$ $g(p, q, r)+3$ since one new sequence appears in each step.

\subsection{The general case}

Denote by $S B_{n}(g)$ the set of members in the $n$-dimensional Stern-Brocot tree of generation $g$, by $S B_{n}^{*}(g)$ the set of members of at most generation $g$, and by $S B_{n}$ the entire tree of dimension $n$.

Denote also by $1_{k}$ an $n$-dimensional vector with all zeroes except one single 1 in the $k$ :th position. The set $1_{1}, \ldots, 1_{n}$ is an ON-base for $\mathbf{R}^{n}$, as well as generation 0 in the tree, and the anchestor set $B_{0}$ for any relatively prime sequence.

Given a sequence $\left(p_{1}, \ldots, p_{n}\right)$ of positive integers with no common multiple, the algorithm starts with the multisequence $\left(p_{1} 1_{1}, \ldots, p_{n} 1_{n}\right)$. Consider a general case $\left(q_{1} P_{1}, \ldots, q_{d} P_{d}\right)$, where $d \leq n$ and $P_{1}, \ldots, P_{d}$ are sequences constituting the ancestor set $B_{i-1}\left(p_{1}, \ldots, p_{n}\right)$. The following algorithm provides the next ancestor set $B_{i}$, in which the leading element is $L_{i}=P_{1}+\ldots+P_{d}$.

Definition 1. Suppose without loss of generality that $q_{1} \leq \ldots \leq q_{d}$. Then a general step of the generating algorithm is defined as follows:

$$
\begin{gathered}
\left(q_{1} P_{1}, \ldots, q_{d} P_{d}\right) \rightarrow \\
\left(\begin{array}{cc}
\left(q_{1}\left(P_{1}+\ldots+P_{d}\right),\left(q_{2}-q_{1}\right) P_{2}, \ldots,\left(q_{d}-q_{1}\right) P_{d}\right) & \text { if } q_{1}<q_{2} \\
\left(q_{1}\left(P_{1}+\ldots+P_{d}\right),\left(q_{3}-q_{1}\right) P_{3}, \ldots,\left(q_{d}-q_{1}\right) P_{d}\right) & \text { if } q_{1}=q_{2}<q_{3} \\
\vdots & \vdots \\
\left(q_{1}\left(P_{1}+\ldots+P_{d}\right),\left(q_{k}-q_{1}\right) P_{k}, \ldots,\left(q_{d}-q_{1}\right) P_{d}\right) & \text { if } q_{1}=\ldots=q_{k-1}<q_{k} \\
\vdots & \vdots \\
\left(q_{1}\left(P_{1}+\ldots P_{d}\right),\left(q_{d}-q_{1}\right) P_{d}\right) & \text { if } q_{1}=\ldots=q_{d-1}<q_{d} \\
\left(p_{1}, \ldots, p_{n}\right) & \text { if } q_{1}=\ldots=q_{d}=1 .
\end{array} .\right.
\end{gathered}
$$

Note that the total sum of the sequences including factors is preserved in the step. A sequence $P$ that can be reached by $g$ steps from the ON-base by this algorithm belong to generation $g$ in $S B_{n}$, written $P \in S B_{n}(g)$, and 
$P_{1}, \ldots, P_{d}$ are its parents. We will later prove that $g$ and the set of parents are well-defined for each relatively prime sequence $\left(p_{1}, \ldots, p_{n}\right)$ (Theorem 1$)$.

The branch $B\left(p_{1}, \ldots, p_{n}\right)=\cup_{i=0}^{g} B_{i}$ consists of all sequences that appear during all steps of the algorithm, i.e. all ancestors of $\left(p_{1}, \ldots, p_{n}\right)$. We will in Theorem 4 find successive direction approximations to $\left(p_{1}, \ldots, p_{n}\right)$ in this set. Note also that $\left|B\left(p_{1}, \ldots, p_{n}\right)\right|=g\left(p_{1}, \ldots, p_{n}\right)+n$ and that

$$
\begin{aligned}
\left(p_{1}, \ldots, p_{n}\right) & =\sum_{P_{j} \in B_{i}} a_{j} P_{j} \text { and } \\
L_{i+1} & =\sum_{P_{j} \in B_{i}} P_{j} .
\end{aligned}
$$

for some positive integers $a_{j}$.

The degree $d \leq n$ of a sequence is the number of parents. It is practical to refer to the part of the tree consisting of entries with full degree the SternBrocot core, while its complement is the pheriphery. Note that the core is an $n$-ary tree with $n^{g-1}$ entries at generation $g$, and that for $n=2$ the Stern-Brocot tree has no pheriphery.

We call a set of parents to a prime sequence a Stern-Brocot set, generalizing the Stern-Brocot pairs of [24]. The ancestor sets are Stern-Brocot sets that are connected to the branch that ends with $\left(p_{1}, \ldots, p_{n}\right)$. Each SternBrocot set $\left\{P_{1}, \ldots, P_{d}\right\}$ spans a $d$-dimensional open convex subset $S\left(P_{1}, \ldots, P_{d}\right)=$ $\left\{c_{1} P_{1}+\ldots+c_{d} P_{d} ; c_{i} \in \mathbf{R}, c_{i}>0, i: 1 \leq i \leq d\right\}$ of $\mathbf{R}^{n}$, containing all further generations of the tree from here. Note further that the Stern-Brocot sets of $S B_{n}(g)$ together with the one-dimensional sets $S(Q), Q \in S B_{n-1}(g)$ provide a partition of the set $\mathbf{R}_{+}^{n}=\left\{\left(x_{1}, \ldots, x_{n}\right) ; x_{i}>0,1 \leq i \leq n\right\}$ in subsets of dimensions $1, \ldots, n$. Denote such a partition by $S P_{n}(g)$, and denote

$$
S P_{n}=\left\{S P_{n}(1), S P_{n}(2), \ldots\right\}
$$

We next provide a general definition to describe this situation.

Definition 2. A multiresolution partition is a sequence of partitions $\Pi=$ $\left\{\pi_{1}, \pi_{2}, \pi_{3}, \ldots\right\}$, where each $\pi_{i}$ is a partition of a fixed set $\Omega$, so that each block in $\pi_{i}$ is the union of at least two blocks in $\pi_{i+1}$.

A multiresolution path of $\Pi$ is a sequence of blocks $s_{1}, \ldots, s_{g}$ where for all $i: 1 \leq i \leq g$ we have $s_{i} \in \pi_{i} \in \Pi$ and $s_{i+1} \subset s_{i}$. 
A multiresolution partition is a natural concept in wavelet analysis. In [36] it is used for earthquake analysis. Certainly a multiresolution path can also be an infinite sequences of sets, for example when considering irrational numbers instead of rationals or integers (page 24).

Obviously, $S P_{n}$ is a multiresolution partition of $\mathbf{R}_{+}^{n}$. The number of blocks for each $g$ can be found by the formula in Theorem 13. The conic hulls of the ancestor sets form a multiresolution path. The Stern-Brocot intervals [18], [15], [38] is $S P_{2}$.

For each ancestor set $B_{i}\left(p_{1}, \ldots, p_{n}\right)$, the vector $\left(p_{1}, \ldots, p_{n}\right)$ belongs to the relative interior of $S\left(B_{i}\left(p_{1}, \ldots, p_{n}\right)\right)$. This means, together with that $S P_{n}(g+$ 1) partitions $S P_{n}(g)$, that the algorithm can never re-enter a block it has left. For each $\left(p_{1}, \ldots, p_{n}\right)$ there is therefore only one sequence of anchestors leading to $\left(p_{1}, \ldots, p_{n}\right)$. It follows that $g$ and the set of parents are well defined.

Theorem 1. Each prime sequence has a well defined set of parents and generation number.

We next need a definition to describe the development of the factor sets.

Definition 3. Consider a multiset of positive integers $\left\{a_{1}, a_{2}, \ldots, a_{n}\right\}$ with at least two distinct integers. Denote $a_{m}=\min \left\{a_{1}, a_{2}, \ldots, a_{n}\right\}$ and $a_{l}=$ $\min \left\{a_{j}: a_{j}>a_{m}\right\}$. We define an extended Euclidan algorithm with the general step

$$
\left\{a_{1}, a_{2}, \ldots, a_{n}\right\} \rightarrow\left\{a_{m}\right\} \cup\left\{a_{j}-k a_{m}: a_{j}-k a_{m}>0, a_{j} \in\left\{a_{1}, a_{2}, \ldots, a_{n}\right\}\right\}
$$

for any $k: 1 \leq k \leq\left\lceil a_{l} / a_{m}\right\rceil-1$.

If $n=2$ and if we choose $k=\left\lceil a_{l} / a_{m}\right\rceil-1$, the algorithm is the usual Euclidean algorithm. Let's say that it is a weak Euclidean algorithm if we constantly keep $k=1$.

Lemma 2. The extended Euclidean algorithm reaches eventually a multiset with only one distinct integer. That integer is $S G D\left\{a_{1}, \ldots, a_{n}\right\}$.

Proof. If the multiset contains at least two distinct positive integers, another step of the algorithm can be performed. Each step strictly decreases the total sum. Hence, we must finally reach a situation with only one distinct integer $A$. If all numbers $a_{1}, \ldots, a_{n}$ are divisible by $m$, then also $A$ is divisible by $m$ 
since then all quantities $a_{j}-k a_{m}$ are divisible by $m$. This argument goes also backwards, so $A=S G D\left\{a_{1}, \ldots, a_{n}\right\}$.

We may consider the spheric surface

$$
S_{+}^{n-1}=\left\{\left(x_{1}, \ldots, x_{n}\right) ; \sqrt{x_{1}^{2}+\ldots+x_{n}^{2}}=1, x_{i} \geq 0 \text { for all } i\right\}
$$

instead of $\mathbf{R}_{+}^{n}$ as a set being partitioned by the tree, by projecting $\left(p_{1}, \ldots, p_{n}\right)$ to $\left(p_{1}, \ldots, p_{n}\right) / \sqrt{p_{1}^{2}+\ldots+p_{n}^{2}}$, i.e. considering normalized vectors. It is consistent with that each prime sequence $\left(p_{1}, \ldots, p_{n}\right)$ represents a unique direction in $\mathbf{R}^{n}$. When projected on $S_{+}^{n-1}$, the multiresolution partition $S P_{n}$ is a set of tesselations in spherical polygons with dimensions from 0 to $n-1$.

We can now summarize main results in this section that follows from the previous considerations. They are completed by Theorem 8 in the next section, that establishes that all generated sequences are relatively prime.

Theorem 3. The algoritm in Definition 1 is for the sets of factors a weak extended Euclidean algorithm, while simultaneously the conic hulls $S\left(B_{i}\right)$ form a multiresolution path in $\mathbf{R}_{+}^{n}$, always containing $\left(p_{1}, \ldots, p_{n}\right)$, and ending in $\left(p_{1}, \ldots, p_{n}\right)$. Also, $S P_{n}$ is a multiresolition partition on $\mathbf{R}_{+}^{n}$, alternatively on $S_{+}^{n-1}$ if considering normalized vectors.

The only purpose of using a weak Euclidean algorithm is to find all ancestors. In the example (page 2.1), Step 5 and the ancestor $(6,3,2)$ would have been dropped if the Euclidean algorithm were not weak.

The following figures show the first generations in the 3-dimensional Stern-Brocot hedge (see page 8 ), so permuting these seqences provide all entries in the tree. Let's denote by $S H_{n}^{*}(g)$ the $n$-dimensional hedge up to generation $g$. In the figures, the members of the last generation are bold. For clarity, only the triangulation for $g=3$ is marked in the graphics of $S_{3}^{*}(3)$ and $S H_{3}^{*}(4)$. 

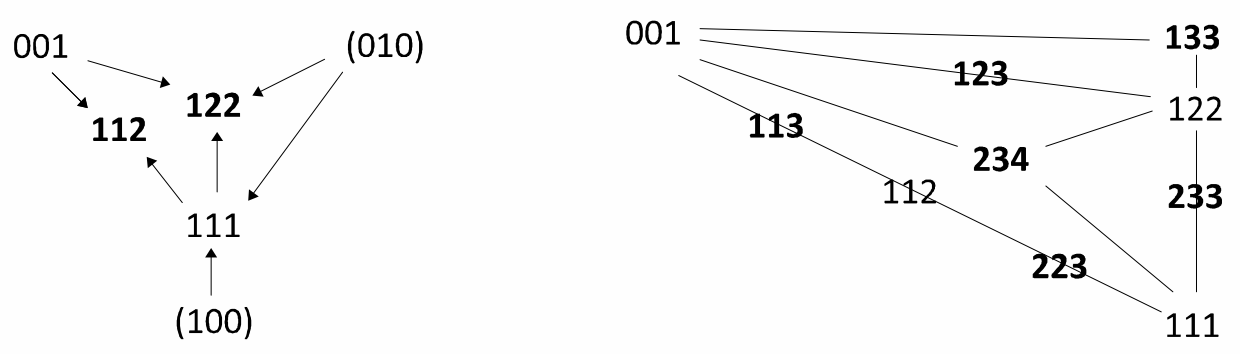

Fig. 2. $S H_{3}^{*}(2)$ and $S H_{3}^{*}(3)$.

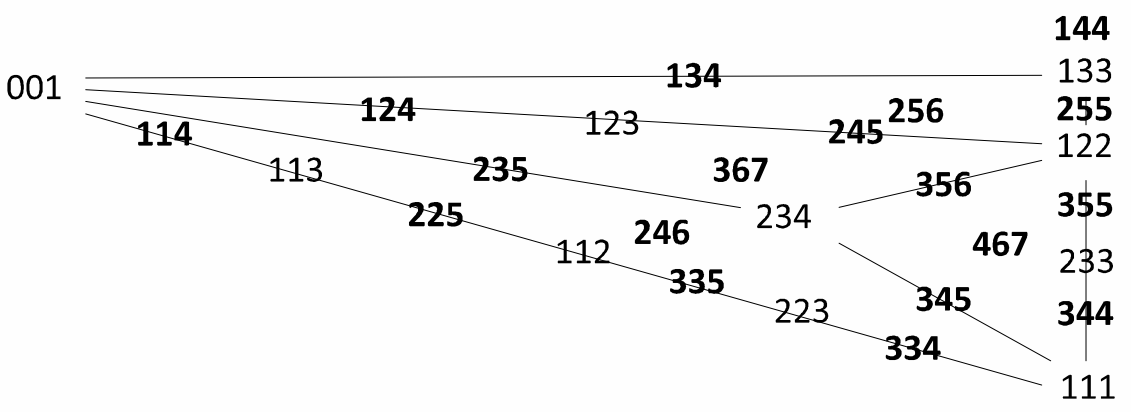

Fig. 3. $S H_{3}^{*}(4)$.

Denote by $\theta(P, Q)$ the angle between the vectors $P$ and $Q$ :

$$
\theta(P, Q)=\arccos \frac{P \cdot Q}{|P||Q|},
$$

where $P \cdot Q=p_{1} q_{1}+\ldots+p_{n} q_{n}$, which we use as a measure of the direction error. Then the leading element of the next generation $L_{i+1}$, i.e, the sum of the entries in $B_{i}$ is a convenient direction approximation of $P=\left(p_{1}, \ldots, p_{n}\right)$. If $d$ is the degree of $L_{i+1}$, the maximal direction error is found by checking $d$ quantities:

Theorem 4. The maximal direction error of $L_{i+1}$ as an approximation of $P$ is

$$
\theta\left(P, L_{i+1}\right) \leq \max _{Q \in B_{i}} \theta\left(Q, L_{i+1}\right)
$$


Proof. In this proof we work on $S_{+}^{n-1}$, i.e. we assume that all vectors are normalized. Denote the boundary of $S\left(B_{i}\right)$ by $\partial S\left(B_{i}\right)$. The set $S\left(B_{i}\right)$ is convex, so all $Q \in S\left(B_{i}\right)$ can be described as $Q=x L_{i+1}+(1-x) R$ for some $R \in \partial S\left(B_{i}\right)$ and $x: 0 \leq x \leq 1$. Then

$$
\begin{aligned}
L_{i+1} \cdot Q & =L_{i+1} \cdot\left(x L_{i+1}+(1-x) R\right) \\
& =x\left|L_{i+1}\right|^{2}+(1-x) L_{i+1} \cdot R
\end{aligned}
$$

is minimal for $x=1$ since $1=\left|L_{i+1}\right|^{2} \geq L_{i+1} \cdot R$. Hence $\theta\left(L_{i+1}, Q\right)$ is maximal for $x=1$, i.e for $Q=R$ at the boundary. Now $R=y L_{i+11}+(1-y) L_{i+12}$ for some $L_{i+11}$ and $L_{i+12}$ in $B_{i}$. If $L_{i+1} \cdot L_{i+11}>L_{i+1} \cdot L_{i+12}$ we get maximal $L_{i+1} \cdot R$ for $y=1$ and $R=L_{i+11}$ alternatively $R=L_{i+12}$ if $L_{i+1} \cdot L_{i+11}<L_{i+1} \cdot L_{i+12}$. In any case, the maximal value of $L_{i+1} \cdot Q$ for any $Q \in S\left(B_{i}\right)$ occurs for $Q \in B_{i}$. Since $P \in S\left(B_{i}\right)$, the theorem follows.

The theorem provides an $n$-dimensional search algorithm to find a prime sequence that approximates the direction of $\left(p_{1}, \ldots, p_{n}\right)$ with a pre-stipulated maximal error.

Each step in the Stern-Brocot core divides an $n$-dimensional polygon in $n$ subpolygons by a point in the interior of the polygon, so each new cell has only one $(n-1)$-dimensional boundary side that is common to the previous polygon. The new sides contain no points in the pheripery, but the single "old" side contains at least one, which is not part of $B_{i}$. The same observation is valid on the relative boundary for lower dimensional polygons.

One may expect that the best approximation of $P$ among the entire $g$ :th generation in the tree could be found among the ancestors. This is true for $n=2$, but not for $n=3$. We will reach a specific cell when searching for any point $P$ inside the cell. So if $P$ is close to an entry not in $B_{i}$ on the "old" side as mentioned above, we have a counterexample.

If including these points to an extended set of ancestors $\bar{B}_{i}=S B_{n}(i) \cap \bar{S}_{i}$, will we in $\bar{B}_{i}$ always find a best approximation for $P$ in $S B_{n}^{*}(i)$ ? This is neither proved or disproved for $n>2$.

Conjecture 5. There is a $Q \in \bar{B}_{i}$ so that $\theta(P, Q) \leq \theta(P, R)$ for all $R \in$ $S B_{n}^{*}(i)$.

In the next section we describe the tree generation-wise, and not branchwise, hence without factors. 


\section{Matrix representations}

Later in this section we will represent each relatively prime sequence by a matrix where the columns are exactly the Stern-Brocot set of the sequence (reduced matrices, see page 19). However, we start with a different representation: in quadratic matrices.

Each relatively prime sequence $\left(p_{1}, \ldots, p_{n}\right)$ of degree $d$ may be represented by a $n \times n$ integer matrix $M$ together with a $d$-partition $D$ of the columns of $M$, i.e. a $d$-partition of the set $\{1,2, \ldots, n\}$. So, $D$ is a set of $d$ non-empty disjoint blocks whose union is $D$. The parents of a sequence are the sums of the columns in each block. This is the central property of the matrix: the block sum property. Hence, the sum of all columns provide the sequence itself. However, the columns in a block are in general not the parents of the sequence represented by that block sum.

We may thus talk about a pair $M, D$, equivalently to a relatively prime sequence. The partition has $|D|=n$ if an only if the sequence is in the core, and then each column is a parent. We assume that the sequence $(1,1, \ldots, 1)$ correspond to the $n$-dimensional unit matrix. In the following the relevance and correctness of this setup are proven.

Let us consider a step in the algorithm in matrix version, from $M$ to $M^{\prime}$, and from partitions $D$ to $E$, where we assume $|D|=d$ and $|E|=$ $e$. Then it follows from the algorithm that the leading element in $M^{\prime}$ is the entire column sum of $M$. According to the algorithm, there is a set of sequences which remain unchanged - these are parents in the next step. There is also a non-empty set of vanishing sequences, that will not be parents. Let $E(D)$ denote the union of all blocks in $D$ that represent vanishing columns. Denote by $D^{\prime}$ the set of remaining blocks in $D$. Then we will preserve the block sum property if all columns in $D-E(D)$ are added to one particular column in $E(D)$ - this provides the matrix $M^{\prime}$. Then $E(D)$ corresponds to the leading element of $M^{\prime}$ since the block sum of $E(D)$ provides the sequence represented by $M$. It is easy to check that the matrix $M^{\prime}$ with the partition $E=\{E(D)\} \cup D^{\prime}$, provides the correct block sums. Then it also has the correct sum over all columns.

Thus the partition $E$ is a specific type of recoarsement of the partition $D$ (more common terminology is that $D$ is a refinement of $E$ ), where one block in $E$ is the union of a set of blocks in $D$, and all other blocks are identical in the two partitions. It follows that there are $\left(\begin{array}{c}d \\ e-1\end{array}\right)$ different partitions with $e$ blocks of this type, $e \leq d$. 
The column sums can be achieved by right-multiplication with certain matrices $I_{i, j}$, for which we assume that $i \neq j$. We assume that the matrix $I_{i, j}$ is a unit matrix except in position $(i, j)$, where it has 1 . Obviously, $\operatorname{det} I_{i, j}=1$, and the matrix $M I_{i, j}$ is identical to $M$ except that the $i$ :th column of $M$ is added to its $j$ :th column. Using such matrices we can define a transition matrix corresponding to the set $E(D)$, that represents the change from $D$ to $E$. Pick a $j \in E(D)$, and define a transition matrix as

$$
I_{E(D)}=\prod_{i \notin E(D)} I_{i, j}
$$

Then $M^{\prime}=M I_{E(D)}$ has the property that all columns in $D-E(D)$ are added to one particular column in $E(D)$. Furthermore, $M^{\prime}$ has determinant 1 by the determinant theorem, and because each matrix $M$ is the product of transition matrices. We have proved the following lemma:

Lemma 6. Let $M, D$ be the matrix of a sequence, and assume that $E(D)$ is the union of the set of blocks in $D$ that correspond to sequences that are not parents to the next sequence. Denote by $D^{\prime}$ the remaining set of blocks in $D$, and $E=\{E(D)\} \cup D^{\prime}$. Pick $j \in E(D)$ and let $I_{E(D)}$ be a transition matrix of $E(D)$. Then $M^{\prime}=M I_{E(D)}, E$ is the matrix corresponding to a child of the sequence whose parents are the block sums of E. It also follows that all matrices have determinant 1.

We thus have recreated the algorithm in matrix form without the factors that govern the algorithm towards a specific branch. The matrix $I_{E(D)}$ is not unique if $|E(D)|>1$ since there are $|E(D)|$ possible indicies $j$ in $E_{k}$ that can be used, so the matrix representation is not unique.

A transition matrix is identical to a unit matrix except that it in one column has from 1 to $n$ :s. Thus, the column sums of these matrices are a certain set of $1: \mathrm{s}$ and $2: \mathrm{s}$, so the set of transition matrices is identical to the second generation of the tree. There are in total $2^{n}-2$ such matrices (sequences). The second generation core has a matrix whith only 1:s in one entire column, so the core has $n$ members in generation 2 . These are the transition matrices for the core.

To prove that the tree contains all relatively prime sequences, and nothing else, we first we need a simple lemma.

Lemma 7. The integers $p_{1}, \ldots, p_{k}$ are relatively prime if and only if there exists integers $a_{1}, \ldots, a_{k}$ so that $a_{1} p_{1}+\ldots+a_{k} p_{k}=1$. 
Proof. If $\left(p_{1}, \ldots, p_{k}\right)$ are not relatively prime there clearly cannot exist such numbers $a_{1}, \ldots, a_{k}$. We prove the other direction by induction over $k$. The statement is well known for $k=2$, and suppose that the statement is true for $k-1$. For $k \geq 3$, if $s=G C D\left(p_{1}, \ldots, p_{. k-1}\right)$ we have numbers $a_{1}, \ldots, a_{k-1}$ so that $a_{1} p_{1}+\ldots+a_{k-1} p_{k-1}=s$ by the induction hypothesis. We also have $G C D\left(s, p_{k}\right)=1$ by the assumption that $p_{1}, \ldots, p_{k}$ are relatively prime. So there are integers $b_{1}$ and $b_{2}$ so that $b_{1} s+b_{2} p_{k}=1$. Inserting $s=a_{1} p_{1}+\ldots+$ $a_{k-1} p_{k-1}$ into this equation completes the induction.

Theorem 8. All sequences $\left(p_{1}, \ldots, p_{n}\right)$ that appear in the $n$-dimensional SternBrocot tree are relatively prime, and all relatively prime $n$-sequences appear.

Proof. The second statment is obvious, since we can start the algorithm with any relatively prime sequence - this is how the tree is defined.

To prove the first statement, we note that if $x_{1}, \ldots, x_{n}$ is a row or a column in an integer matrix with determinant 1 , subdeterminants provide integers $a_{1}, \ldots, a_{k}$ so that $a_{1} x_{1}+\ldots+a_{n} x_{n}=1$. But every sequence in the core occurs as a column in the matrix representation of its children, and all matrices have determinant one, so by Lemma 7 all sequences in the core are relatively prime. By construction, sequences in the periphery occur as sums of columns. Since the value of a determinant does not change if a column is added to another column, column additions can be repeated until a sequence in the pheripery occur as a single row in a matrix of determinant 1 . Thus also the sequences in the pheriphery are relatively prime.

Not only the sequences are relatively prime. We can also prove that all appearing sets of factors are relatively prime.

Lemma 9. All sets of factors $\left(q_{1}, \ldots, q_{d}\right)$ that appear in the algorithm, starting with a relatively prime sequence $\left(p_{1}, \ldots, p_{n}\right)$, are relatively prime.

Proof. This is easily is proven by induction over the steps of the algorithm. In the start we have $\left(q_{1}, \ldots, q_{n}\right)=\left(p_{1}, \ldots, p_{n}\right)$, so the statement is true. If $G C D\left(q_{1}, \ldots, q_{d}\right)=1$ and $q_{1}=\ldots q_{d^{\prime}-1}<q_{d^{\prime}}$, then $G C D\left(q_{1}, q_{d^{\prime}}-q_{1}, \ldots, q_{d}-\right.$ $\left.q_{1}\right)=s>1$ is impossible by a simple application of Lemma 7 . The lemma is proven.

Lemma 10. A sequence of degree $d$ has $\left(\begin{array}{c}d \\ e-1\end{array}\right)$ children of degree e, and in total $2^{d}-2$ children. 
Proof. We have seen before Theorem 6 that to a multisequence $P_{1}, \ldots, P_{d}$, in matrix form $M$ with a partition $D$, there are $\left(\begin{array}{c}d \\ e-1\end{array}\right)$ different partitions potentially providing a child of degree $e$. We need also to check that all $\left(\begin{array}{c}d \\ e-1\end{array}\right)$ partitions may occur in the algorithm. Choose the factors $q_{1}, \ldots, q_{d}$ so that a specific set of $d-e+1$ factors are equal, and thus give rise to a partition of $e$ blocks. Running the algorithm backwards and using the argument in the proof of Lemma 9 , we will end with the multisequence $x_{1} 1_{1}, \ldots, x_{n} 1_{n}$ where $x_{1}, \ldots, x_{n}$ are relatively prime. Hence, there is a relatively prime sequence that provides each child of $P_{1}, \ldots, P_{d}$ that corresponds to each possible partition.

Adding the number of partitions over $e$ provides the sum $2^{d}-1$. However, the partition with one single block has a block sum that is identical to the sequence itself, and is thus not a child.

For a sequence $\left(p_{1}, \ldots, p_{n}\right)$ with parents $P_{1}, \ldots, P_{d}$, any sum of $k$ parents, $1 \leq k \leq d$ provides a direction that does not leave the conic hull $S\left(P_{1}, \ldots, P_{d}\right)$, i.e. each such parent sum may be added to $\left(p_{1}, \ldots, p_{n}\right)$. In matrix terms this can be seen as multiplication by a certain subset of the second generation.

The next theorem specifies the allowed subset of generation 2 . If $P$ is a sequence and $D$ a partition, denote by $D(P)$ the entries in $P$ divided in blocks according to the partition $D$.

Theorem 11. Suppose a sequence of degree $d$ is represented by a matrix with a partition D. Allowed matrices of the second generation is then those corresponding to a sequence $P$ of 1:s and 2:s so that all blocks in $D(P)$ contains only 1:s or only 2:s.

Proof. This respects the only rule of matrix multiplication, that each block should either not be addedd to another column at all, or the entire block should be added.

For example, the development after the sequence $(1,1,2)$ of degree 2 in $S B_{3}$ that has partition $D=\{\{1,2\},\{3\}\}$, the only allowed products are with the matrices corresponding to $(2,2,1)$ and $(1,1,2)$. Non-allowed products, i.e. containing a mixed block, may be possible and useful, but they do not support the tree structure or the algorithm that defines the generations of the tree, as expressed by Theorem 1 .

The second generation can be regarded as a set of directions in which the next step may be taken without leavning the conic hull. Roughly, each member $P$ of $S B_{n}(2)$ moves by right matrix multiplication the sequence in a direction towards $P-1_{n}$. In this description we allow sums of the ON-bases 
that have less than $n$ terms, i.e. members of the Stern-Brocot grove (see page 8 ). While the tree hs one single member of generation 1 , the grove has $2^{n}-n-1$ members.

Our next purpose is to establish the topological result of Theorem 12, comparing a subtree with an entire tree. Let $M, D \in S B_{n}$, and denote by $S B_{n}(M, D)$, the subtree of $S B_{n}$ that consists of all descendants of $M, D$. For this tree we define a relative generation number counting from $M, D$, so $g(M, D)=1$.

Denote the reduced matrix $\bar{M}$ to a sequence to be the matrix of type $n \times d$ where, simply, each column is a parent. Then, $\bar{M}=M$ if and only if $M$ belongs to the core, and the sequence is still the sum of all columns. For a partition $D=\left\{D_{1}, \ldots, D_{d}\right\}$, denote the $i$ :th block in $M$ by $M_{i}$. Then if $I_{D}$ is a $n \times d 0,1$-matrix with $I_{D}(i, j)=1$ if and only if $M_{i} \in D_{j}$, then $\bar{M}=M I_{D}$.

Thus $S B_{n}$ can be described in three ways: as a tree of sequences, as a tree of $n \times n$ matrices each with an associated partition, or as a tree of reduced matrices.

Let $M$ be a reduced matrix of type $n \times d$. Then the Moore-Penrose pseudoinverse $M^{+}$is a left inverse to $M$, so $M^{+} M=E_{d}$, if we denote the $d \times d$ unit matrix by $E_{d}$. Note that also $M E_{d}=M$, so we may say that left multiplication with $M^{+}$maps from $S B_{n}(M)$ to $S B_{d}$, and left multiplication with $M$ maps from $S B_{d}$ to $S B_{n}(M)$.

We can now observe the following:

Theorem 12. Let $M$ be the matrix of a relatively prime sequence with degree d. Then the subtree $S B_{n}(M)$ is isomorphic to $S B_{d}$ in the sense that

1. $A$ is a parent of $B$ in $S B_{n}(M)$ if and only if $M^{+} A$ is a parent of $M^{+} B$ in $S B_{d}$.

2. $A$ is a parent of $B$ in $S B_{d}$ if and only if $M A$ is a parent of $M B$ in $S B_{n}(M)$.

The trees $S B_{n}(M)$ and $S B_{d}$ have the same number of members at each generation $g$.

Proof. Assume that $A$ is a parent of $B$ in $S B_{n}(M)$, and assume that $A$ and $B$ are reduced matrices. If $A$ and $B$ are the same degree, then it follows that 
$B I_{D}=A$ where $I_{D}$ is a member of $S B_{d}(2)$, simply by considering column sums in $B I_{D}$. Then obviously $\left(M^{+} B\right) I_{D}=M^{+}\left(B I_{D}\right)=M^{+} A$, so $M^{+} A$ is a parent of $M^{+} B$. If $d(A)>d(B)=e$, the same argument is true if we use a reduced version $\bar{I}_{E}$ of type $d \times e$. The proof of 2 . is similar.

\subsection{Combinatorial properties}

We next check some fundamental combinatorics for $S B_{n}$ in terms of the number of sequences in each generation, and fastest growing branches.

Denote by $T_{n}(g)$ the number of sequences in generation $g$ in the $n$ dimensional Stern-Brocot tree. The following formula generalizes $T_{2}(g)=$ $2^{g-1}$ and $T_{n}(2)=2^{n}-2$.

Theorem 13. $T_{n}(0)=n, T_{n}(1)=1$, and for $g \geq 2$ we have

$T_{n}(g)=2(-1)^{g-1}+\sum_{k=2}^{g} k^{n}\left(2(-1)^{g-1}+\sum_{j=1}^{k-1}\left(\begin{array}{c}k-1 \\ j\end{array}\right)(j+2)(-(j+1))^{g-2}(-1)^{j-1}\right)$.

Proof. For $g \leq 1$, we observe that generation 0 is the ON-base, and generation 1 consists only of $(1, \ldots, 1)$.

Assume that $g>1$. In $S B_{n}(2)$ we have $\left(\begin{array}{c}n \\ k-1\end{array}\right)$ sequences that contain $k-1$ 2 :s and $n-k+11$ :s. Each set containing $(1,1, \ldots, 1)$ and a selection of $k-1$ unit vectors provides a start for a tree that by Theorem 12 is topologically equivalent to $S B_{k}$. Hence each of them produce the same number of sequences, and we obtain a recursion for the entire tree $S B_{n}$. Since there are $\left(\begin{array}{c}n \\ k-1\end{array}\right)$ such sets, we have the recursion $T_{n}(g)=\sum_{k=2}^{n}\left(\begin{array}{c}n \\ k-1\end{array}\right) T_{k}(g-1)$. It is easy to check that the formula in the theorem gives $T_{n}(2)=2^{n}-2$. The proof of induction is then a matter of pure calculation by inserting the formula in the right hand side of the recursion, taking advantage of $\sum_{i=2}^{n}\left(\begin{array}{c}n \\ i-1\end{array}\right) k^{i}=k\left((k+1)^{n}-k^{n}-1\right)$ for all $k \geq 1$.

Next we describe a subset of the core that provide the fastest growing sequence sums in the tree.

Definition 4. A sequence of generation $g \geq 1$ in the core is a Fibonacci type sequence if the $n$ parents belong to generations $\max (g-i, 0)$ for $i=1, \ldots, n$.

If we denote the parent of $\left(p_{1}, \ldots, p_{n}\right)$ of generation $\max (g-j, 0)$ by $\left(p_{1, g-j}, \ldots, p_{n, g-j}\right), 1 \leq j \leq n$, Fibonacci type sequences fulfill $p_{k, g}=p_{k, g-1}+$ 
$\ldots+p_{k, g-n}$ for all $1 \leq k \leq n$, assuming that $p_{k, g}=0$ for all $k$ if $g \leq 0$, except that for each $g \leq 0$ there is one $k$ where $p_{k, g}=1$.

This is similar to the Fibonacci $n$-step sequence $\left\{F_{1}^{(n)}\right\}_{k=1}^{\infty}$, that is defined as $F_{k+1}^{(n)}=F_{k}^{(n)}+\ldots+F_{k-n+1}^{(n)}$ with initial values $F_{1-n}^{(n)}=1$ and $F_{k}^{(n)}=0$ for $k=-n+2, \ldots, 0$ [29]. Compared to the Fibonacci $n$-step sequence, sequences of Fibonacci type have exactly $n$ members, they may appear in any permutation (not necessarily increasning order), and may have any ONbase vector as starting values (not necessarily $(0, \ldots, 0,1)$ ). So $n$ consecutive numbers of the Fibonacci $n$-step sequence is a sequence of Fibonacci type.

In [29] the scarcity of prime Fibonacci $n$-step numbers is stressed. However, $n$ consecutive such numbers have never common multiples.

Lemma 14. 1. Any set of $n$ consecutive Fibonacci $n$-step numbers are relatively prime.

2. $\left(p_{1}^{\prime}, \ldots, p_{n}^{\prime}\right) \in S B_{n}(g)$ is Fibonacci type if and only if $p_{1}+\ldots+p_{n} \leq$ $p_{1}^{\prime}+\ldots+p_{n}^{\prime}$ for all $\left(p_{1}, \ldots, p_{n}\right) \in S B_{n}(g)$.

3. The number of sequences of Fibonacci type at generation $g$ is $\frac{n !}{(n-g+1) !}$ if $g<n$ and $n$ ! if $g \geq n$,

Proof. 1. This follows directly from Theorem 8.

2. This follows by induction. Iff sequences $P_{1}, \ldots, P_{n}$ have the largest sequence sums in generations $\max (0, g-n), \ldots, \max (0, g-1)$ for $g>0$, and form a Stern-Brocot set, then $P_{1}, \ldots, P_{n}$ has the largest sequence sum in generation $g$. This is true for $g=1$, and thus for all $g$, and it is exactly the definition of Fibonacci type sequences.

3. If $g<n$, a Fibonacci type sequence has $n-g+1$ integers that are $2^{g-1}$, hence equal, while the other $g-1$ are distinct from each other and from $2^{g-1}$. Hence the number of permuations is $\frac{n !}{(n-g+1) !}$. If $g \geq n$, all are distinct, so we have $n$ ! permutations.

\section{Conclusions and extensions}

Denote by $S(M) \in \mathbf{R}^{n}$ the convex hull of the column vectors $M_{1}, \ldots, M_{n}$ of the matrix $M$. Next we pick a few definitions of Brentjes formalism [9] on multidimensional continued fractions [31], using the notation of the present paper: 
Definition 5. Consider a finite or infinite sequence of $n \times n$-matrices $M(0), M(1), \ldots$ so that $M_{i}(0) \cdot M_{j}(0) \geq 0$ for all $i, j$, and so that for each $k \geq 0$ there exist integers $s, t$ and $b$ so that $1 \leq s, t \leq n, s \neq t$ and $b \geq 1$ so

$$
M_{i, j}(k+1)=\left\{\begin{array}{cc}
M_{i, j}(k)+b M_{i, s}(k) & \text { if } j=t \\
M_{i, j}(k) & \text { if } j \neq t
\end{array},\right.
$$

and $v_{0} \in S(M(k))$. Then $M(0), M(1), \ldots$ is an $n$-dimensional continued fraction expansion of $M$ along a vector $v_{0} \in \mathbf{R}^{n}$.

Definition 6. An algorithm that produces the sequence $\{M(i)\}_{i}$, and thereby fixes values $s, t$ and $b$, is a multidimensional continued fraction algorithm. It is called subtractive if always $b=1$, and it is called a division algorithm if, denoting $v_{0}=M(i) a(i)$, always $a_{t}(i)>0$ and $b=\left\lfloor a_{s}(i) / a_{t}(i)\right\rfloor$.

The following theorem describes the connection between the $n$-dimensional Stern-Brocot tree to continued fractions. It is included in this section of the paper since it suggests further extensions only.

Theorem 15. A sequence of matrices $M(0), M(1), \ldots$ representing a relatively prime sequence, with $M(0)$ a unit matrix, as generated by the algorithm of Definition 1, is a subsequence of an $n$-dimensional continued fraction expansion of the $O N$-base along $v_{0}=\left(p_{1}, \ldots, p_{n}\right)$.

The algorithm, that by Theorem 3 is governed by a weak extended Euclidean algorithm, is a subtractive $n$-dimensional continued fraction algorithm, and it is not a division algoritm.

Proof. Consider the transition matrix $I_{E(D)}=\prod_{i \notin E(D)} I_{i, j}$ in the proof of Lemma 6. Picking $b=1$ in Definition 5 and including all matrix multiplied by each matrix $I_{i, j}$ at a time provides an $n$-dimensional continued fractal according to the definition, since $v_{0} \in S(M(i))$ is fulfilled for all $i$. Also it is clear that $S\left(M I_{i, j}\right) \subset S(M)$, so the condition $v_{0} \in S(M)$ is true also for all intermediate matrices. Hence the algorithm is an $n$-dimensional subtractive continued fraction algorithm.

The factor sets are the sequences $a_{1}(i), \ldots, a_{n}(i)$ for all $i$, where a factor corresponding to a vector that is represented by a sum of columns in $M$ simply is repeated for all those columns. Thus, $a_{1}, \ldots, a_{n}$ are for the present algoritm all strictly positive, and all equals one in the last step. The entry $a_{t}$ is the smallest entry, but $a_{s}$ are the other entries in turn, so the ratio 
$a_{s} / a_{t}$ may well be 2 or larger, as shown by the Example 2.1. It is thus not a division algorithm by Definition 6 .

There are other variants of multidimensional fractions that are all equivalent [30] - the terms Mönkemeyer algorithm [26], Selmer algorithm [33] and GMA (generalized mediant algorithm) [3] occur. So we have a connection between the Stern-Brocot tree and continued fractions also in $n$ dimensions. This is promising since research in multidimensional continued fractions has seen a lot or progress in the last decades [31], and it has strong relations to for example ergodic thery and Diophantine approximation.

We have seen that it is possible to extend the well known Stern-Brocot tree of pairs to a tree of $n$-tuples, with a similar tree structure and isomorphism of subtrees to the entire tree (Theorem 12). There is however the extra complication of prime sequences having different degrees, forcing the quadratic matrix representation to be completed with a partition. The second generation of the tree can be seen as a generator of the entire tree also for $n>2$. However, the correct subset of this generation need to be used (Theorem 11). It also forces the conic hulls of ancestor sets, containing all subsequent sequences, to have different dimensions. The possible number theoretic significance of the degree of a prime sequence, outside this context, is unknown.

The construction also involves a generalization of the Euclidean algorithm. The two dimensional tree is closely intertwined with the Eucidean algorithm via continued fractions. For $n>2$ we have seen that each branch of the tree can be generated by means of a weak version of an $n$-dimensional Euclidean algorithm, using the goal sequence as starting factors. Simultaneously, the vectors/matrices themselves are not a division algorithm in the terminology of multidimensional continued fractions.

As for $S B_{2}$ the generating algorithm of Theorem 1 can be used to provide simultaneous rational approximations of a set of irrational numbers (see [21] for $n=2) \alpha_{1}, \ldots, \alpha_{n}$. For example, starting with $\left(\alpha_{1} 1_{1}, \ldots, \alpha_{n} 1_{n}, 1_{n+1}\right)$, the leading element $\left(p_{1}, \ldots, p_{n+1}\right)$ provides rational approximations $p_{1} / p_{n+1}, \ldots, p_{n} / p_{n+1}$ for $\alpha_{1}, \ldots, \alpha_{n}$ with a maximal error as described in Theorem 4 .

Conjecture 5 is open. One may also investigate the rate of decrease of the Lebesgue measure of the blocks in $S P_{n}(g+1)$ compared to the blocks in $S P_{n}(g)$ as projections to $S_{+}^{n-1}$. Similar results for the diameter $\max _{x, y \in S}|x-y|$ 
of a block $S$ may improve the error estimate of Theorem 4, however, there are multiresolution paths with constant diameter of the sets. Furthermore, the complexity of the generating algorithm may be investigated.

The results offer possibilities to extend various number theoretical concepts to $n$ dimensions, such as applications described in Subsection 1.2 or interpretations of the $n$-dimensional Minkowski question mark function [30] in terms of the tree, i.e. of relatively prime sequences.

\section{References}

[1] G. Alkauskas, The moments of Minkowski question mark function: the dyadic period function, Glasgow Math. J. 52 (2010), 41-64.

[2] T. Apostol, Modular Functions and Dirichlet Series in Number Theory, Second Edition (1990), Springer, New York.

[3] P. R. Baldwin, A multidimensional continued fraction and some of its statistical properties, J Statist Phys 66, 1463-1505 (1992).

[4] B. Bates, M. Bunder, Locating terms in the Stern-Brocot tree, Eur. J. of Combin. 31(3) (2010), 1020-1033.

[5] B. Bates, M. Bunder, K. Tognetti, Lingages between the Gauss map and the Stern-Brocot tree, Acta Math. Acad. Paedagog. Nyházi 22 (2006) 217-235.

[6] J. Berstel, A. de Luca, Sturmian words, Lyndon words and trees, Theoretical Computer Science 178 (1997), 171-203.

[7] O. Braun, G. Schmidt, Parallel Processor Scheduling with Limited Number of Preemptions, Siam J. of Comput., Vol 32, No. 3 (2003), pp. 671680 .

[8] J. F. Bonnans, E. Ottenwaelter, H. Zidani, A fast algorithm for the two dimensional HJB equation of stocastic control, ESAIM: Math. Model. Numer. Anal. 38 (4) (2004), 723-735.

[9] A. J. Brentje, Multi-dimensional continued fraction algorithms, MAthematical Centre Tracts 145, Amsterdam: Mathematisch Centrum (1981). 
[10] A. Brocot, Calcul des rouages par approximation, nouvelle méthode, Revue Chronométrique 3 (1861), 186-194.

[11] M. Bullejos, J. C. Rosales, Proportionally modular Diothantine inequalities and the Stern-Brocot tree, Math. of Comput. Vol 78, No 266 (2009) 1211-1226.

[12] J. H. E. Cartwright, J. Douthett, D. L. González, Two musical paths to the Farey seris and devil's starcase, J. Math. Music 4(1) (2010), 57-74.

[13] E. G. Coffman, and M. R. Garey, Proof of the 4/3 conjecture for preemptive vs. nonpreemptive two-processor scheduling. J. ACM, 40(5) (1993), 991-1018.

[14] A. Denjoy, Sur une fonction réelles de Minkowski, J. Math. Pures Appl. 17 (1938), 105-151.

[15] M. Embree, L. N: Trefethen, Growth and Decay of Random Fibonacci Sequences, Proceedings: Mathematical, Physical and Engineering Sciences, Vol. 455, No 1987 (1999) 2471-2485.

[16] J. G. Freire, J. A. C. Gallas, Stern-Brocot trees in cascades of mixedmode oscillations and cnadrs in the extended Bonhoeffer-van der Pol and the FitzHugh-Nagumo models of exitable systwems, Physics Letters A 375(7) (2011), 1097-1103.

[17] P. Gibbs, A Generalised Stern-Brocot Tree from Regular Diophantine Quadruples, arXiv:math/9903035v1, Cornell University (1999).

[18] M. Kesselböhmer, B.O. Stratmann, A multifractal analysis for Stenrbrocot intervals, continued fractions and Diophantine growth rates, $J$. für die reine und angewandte Mathematik 605, Vol 2007 (605) (2007), 133-163.

[19] P. Kornerup, D. Matula, An on-line arithmetic unit for bit-pipelined rational arithmetic, J. Parallel Distr. Com. 5 (1988), 310-330.

[20] C. Karanikas, N.D. Atreas, A. Bakalakos, P. Polychronidou, Discrete Transforms and Symbolic Sequences, Scientific Support for the Decision Making in the Security Sector, IOS Press, Velingrad, Bulgaria (2007), 126-137. 
[21] R. Graham, D. Knuth, O. Patashnik, Concrete Mathematics, Addison Wesley, 1994.

[22] L. Lundberg, H. Lennerstad, The maximum gain of increasing the number of preemptions in multiprocessor scheduling, Acta Inform. 46(4) (2009), 285-295.

[23] E. Laksman, H. Lennerstad, M. Nilsson, Improving bounds on the minimal Euclidean distance for block codes by inner metric optimization, Discrete Mathematics 310 (22): 3267-3275 (2010).

[24] H. Lennerstad, L. Lundberg, Decomposing rational numbers, Acta Inform. 145(3) (2010), 213-220.

[25] H. Minkowski, Zur Geometrie der Zahlen, Verhandlungen des III Internationalen Mathematiker Kongressous, Heidelberg 1904, 164-173.

[26] R. Mönkemeyer, Über Fareynetze in $n$ Dimensionen, Math Nacr 11, 321-344 (1954).

[27] M. Niqui, Exact arithmetic on the Stern-Brocot tree, J. of Discrete Algorithms, Vol 5(2), (2007), 356-379.

[28] T. Noll, Music Intervals and Special Linear Transforms, J. of Math. Music 1(2) (2007), 121-137.

[29] Noe T.,Vos Post J, Primes in Fibonacci $n$-step and Lucas $n$-step Sequences, Journal of Integer Sequences, Vol 8 (2005).

[30] G. Panti, Multidimensional continued fractions and a Minkowski function, Monatsh Math. 154, Vol 3, 247-264 (2008).

[31] F. Schweiger, Multidimensional Continued Fractions, Oxford Univ. Press, New York, (2000).

[32] C. Series, The Modular Surface and Continued Fractions, J. London Math. Soc. (1985) s2-31 (1): 69-80.

[33] E. S. Selmer, Continued fractions in several dimensions, Mordisk Nat Tidskr 9, 37-43, 95 (1961). 
[34] M. A. Stern, Über eine zahlentheoretische Funktion, J. Reine Angew. Math. 55 (1858), 193-220.

[35] G. Strang, Wavelets and dilation equations: a brief introduction, SIAM Review 39 (1989), 383-406.

[36] M. L. Todorovska, T.-V. Hao, Representation and Compression of Structural Vibration Monitoring Data Using Wavelets as a Tool in Data Mining, proceedings of 13th World Conference of Earthquake Engineering, Vancouver, Canada, Aug. 1-6, 2004, paper no. 2954.

[37] F. de Vieilleville, J.-O. Lachard, Revisiting Digital Straight Segment Recognition, Discrete Geometry for Computer Imagery, Lecture Notes in Computer Science, Vol 4245/2006 (2006), 355-366.

[38] D. Viswanath, Math. of Comput, 69 (231), (1999), 1131-1155. 
THE N-DIMENSIONAL STERN-BROCOT TREE

\section{HÅKAN LENNERSTAD}

Printed by Printfabriken AB, Karlskrona 2012.

ISSN I 103-158|

ISRN BTH-RES-04/I2-SE 